\title{
Novel Compressed Digital Radio Fronthaul over Photonically-generated THz Wireless Bridge
}

\author{
Tongyun Li1 ${ }^{*}$, Luis Gonzalez-Guerrero ${ }^{2}$, Haymen Shams ${ }^{2}$, Cyril Renaud ${ }^{2}$, Alwyn J. Seeds ${ }^{2}$, Martyn Fice ${ }^{2}$, Ian \\ White ${ }^{1}$, Richard Penty ${ }^{1}$ \\ ${ }^{1}$ Centre for Photonic Systems, Electrical Division, Department of Engineering, University of Cambridge, 9 JJ Thomson Avenue, Cambridge, U.K \\ ${ }^{2}$ Department of Electronic and Electrical Engineering, University College London, London, UK \\ *tl299@cam.ac.uk
}

Abstract: Compressed DRoF-based fronthaul links enable cost-effective last-mile wireless coverage. This paper demonstrates a novel system which carries 12 LTE services over both optical fibre and photonically-generated $\mathrm{THz}$ wireless links with over $40 \mathrm{~dB}$ dynamic range.

\section{Introduction}

Common public radio interface (CRPI) based digital radio over fibre (DRoF) fronthaul is well established for lastmile wireless coverage for $4 \mathrm{G}$ and $5 \mathrm{G}$. However, the increased data rate and low spectral efficiency (e.g. single $20 \mathrm{MHz}$ LTE requires a data rate of $1.25 \mathrm{Gbps}$ ) are major drawbacks causing inefficient utilisation of optical infrastructure deployed [1]. Newly developed evolved CPRI (eCPRI) and radio over Ethernet (RoE) protocols define new functional splits to minimise the transmission line rate between the remote radio unit (RRU) and baseband unit (BBU) [2]. Although the high-layer splits can ultimately reduce the data rate, flexibilities of a common interface are lost because that more vendor specific information is required. In contrast, a compressed DRoF transmission is demonstrated in [3] demonstrating a 3-times higher transmission efficiency compared with CPRI or eCPRI at functional split 8, which is the RF and low PHY interface. A key benefit is that a neutral host (i.e. multi-service multioperator) wireless coverage system can be established based on this architecture without any previous knowledge of the mobile network operators (MNOs) or equipment vendors. To extend the capability of such a system, the compressed digital RF signals can be transmitted over millimetre wave $(\mathrm{mmW})$ carriers enabling a brand-new way to deploy the system by allowing wireless fronthaul links to replace optical fibres [4,5]. This new architecture not only supports multi-service digital RF transmission over the air with high transmission efficiency, but also has a wider input power dynamic range than that of any predecessors in the $\mathrm{mmW}$ band using analogue approaches.

In this paper, we demonstrate a 6 Gbps compressed digital fronthaul transmission carrying twelve $20 \mathrm{MHz}$ LTEcompatible services over both optical fibre and terahertz $(\mathrm{THz})$ wireless links. This is the first demonstration of such a system to our knowledge and could offer a profound change in approach for next-generation multi-service wireless coverage in $5 \mathrm{G}$ and beyond.

\section{System Architecture}

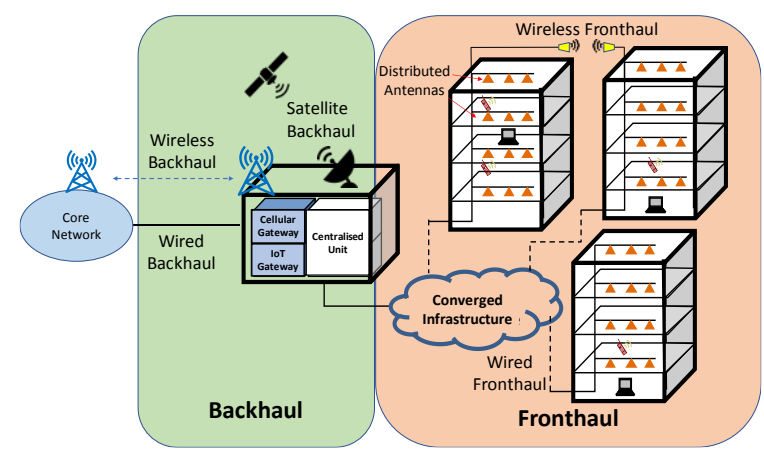

(a)

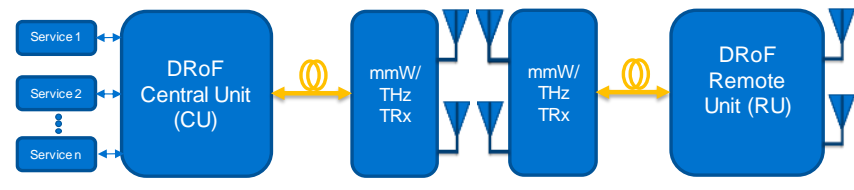

(b)

Fig 1. (a) Heterogeneous wireless coverage system. (b) DRoF with mmW bridge.

The next generation radio access network (RAN) for distributed indoor wireless coverage will be a heterogeneous network (Fig 1(a)) allowing flexible deployment of wireless services and converged network infrastructure. Coexistence of $\mathrm{mmW} / \mathrm{THz}$ based wireless fronthaul and backhaul will enhance cost effectiveness and fast rollout of the network [6]. The system architecture proposed here incorporates both optical and $\mathrm{mmW} / \mathrm{THz}$ wireless transmission and is shown in Fig 1(b). In the downlink, multiple wireless services are digitised, compressed and packetized in the DRoF central unit (CU) before optical transmission. The compressed baseband digital signal is then upconverted to 
the $\mathrm{mmW} / \mathrm{THz}$ band for wireless transmission in a point-to-point link. At the remote unit (RU), the carriers are down converted and subsequently recovered fully to its original analogue RF signal for wireless distribution.

\subsection{DRoF data compression and signal processing}

At the CU, as shown in Fig 2, the RF signal is firstly down converted to an intermediate frequency (IF) located at the centre of the second Nyquist Zone before digitisation by an analogue to digital converter (ADC) in order to relax the performance requirements of the analogue frontend design. Field programmable gate arrays (FPGA) are used to perform digital signal processing upon the digitised IF signal which is then compressed in order to reduce the quantisation bit width through register-transfer level (RTL) design using Verilog. A digital automatic gain controller (DAGC) is implemented on the FPGA to maintain the signal power level before the first compression stage so that the dynamic range of signal is not affected after compression. A digital down conversion (DDC) module moves the signal central frequency to baseband allowing a root raise cosine (RRC) filter to remove spectrum redundancy. The second compression stage decimates the signal sampling rate in the frequency domain to minimise the data rate. The resulting low bit rate data is packetized using the $8 \mathrm{~b} / 10 \mathrm{~b}$ coding scheme with a $\mathrm{k} 28.5$ synchronisation code to allow its word alignment to be restored at the receiver. The signal is fully recovered to digital IF on the receiver FPGA at the RU before digital to analogue conversion (DAC) as depicted in Fig 2.

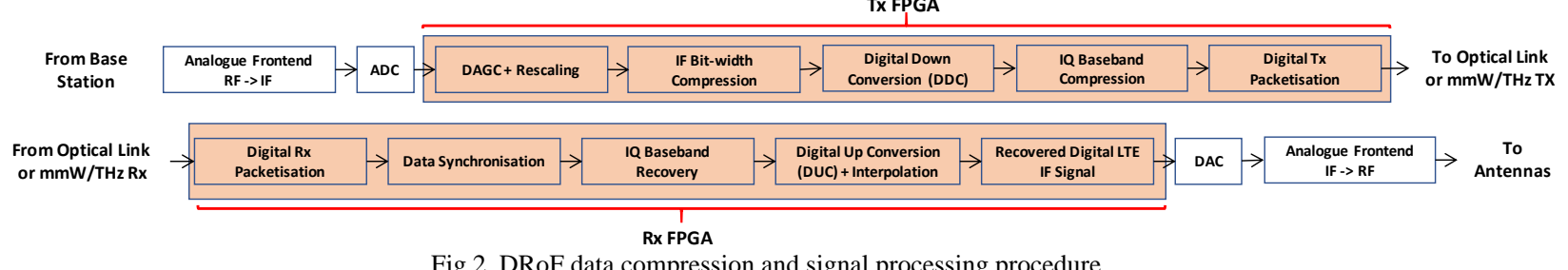

\section{Experimental Setup and Results}

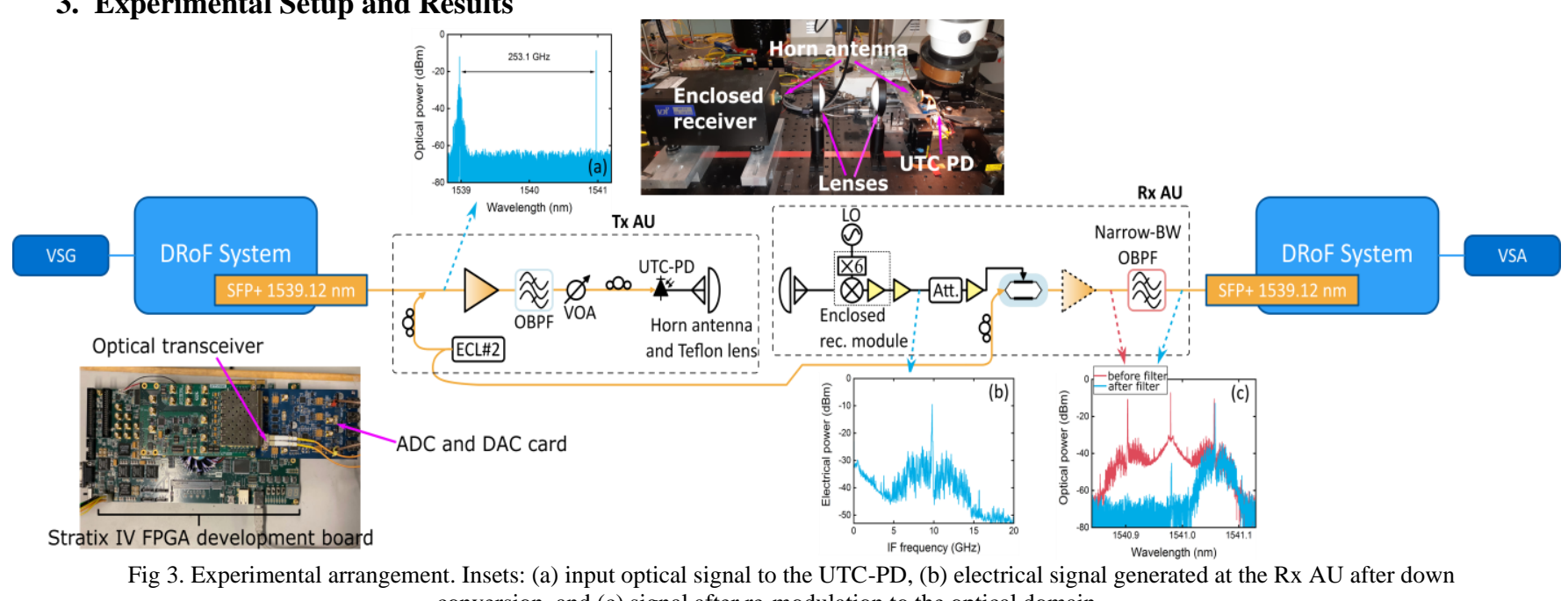
conversion, and (c) signal after re-modulation to the optical domain.

As shown in Fig 3, a $20 \mathrm{MHz}$ LTE signal at a $115.2 \mathrm{MHz}$ IF is generated with a vector signal generator (VSG) and sent to a 14-bit ADC sampling at $150 \mathrm{MHz}$ for digitisation and then to an Altera Stratix IV FPGA where the steps described in Section 2.1 for signal compression and digital signal processing take place. During the process, a resultant $2.1 \mathrm{Gbps}$ digitised data is compressed to $400 \mathrm{Mbps}$ per service. To simulate multi-channel scenario, the input signal is replicated to give 12 parallel processing chains before packetization on to a $6 \mathrm{Gbps}$ serial data frame, allowing some redundancy for data synchronisation. The packetized signal from the DRoF unit is modulated onto a distributed feedback (DFB) laser operating at $1538.19 \mathrm{~nm}$ through an enhanced small form-factor pluggable (SFP+) transceiver. The optical signal from the SFP+ transceiver is then sent to the transmitter antenna unit (Tx AU), where it is combined with the output of an external cavity laser operating at $1540.19 \mathrm{~nm}$ - giving a frequency difference between the two lasers of $253.1 \mathrm{GHz}$ (see Fig. 3 inset (a)). After optical amplification and filtering, the two optical tones are fed into an unpackaged uni-travelling carrier photodiode (UTC-PD) by means of a lensed fibre. Horn antennas with a gain of $25 \mathrm{dBi}$ are used for both transmission and reception of the resultant $\mathrm{THz}$ signal and are placed $0.24 \mathrm{~m}$ apart. A pair of Teflon lenses with a diameter of $5 \mathrm{~cm}$ and a back focal length of $63.2 \mathrm{~mm}$ were inserted between the two antennas (the separation between the lenses was around $10 \mathrm{~cm}$ ) to increase the collimation of the $\mathrm{THz}$ beam. 
At the receiver $(\mathrm{Rx}) \mathrm{AU}$, the signal is down-converted to an IF frequency of around $10 \mathrm{GHz}$ with an enclosed receiver module (WR3.4MixAMC from Virginia Diodes) consisting of a $\times 6$ multiplier, a sub-harmonic mixer (SHM), and an IF amplifier. After down-conversion, the IF signal is passed through two additional IF amplifiers (see Fig. 3). The resultant electrical signal is used to drive an intensity modulator (IM) which is biased at the null point to supress the optical carrier (see Fig. 3 inset (c)). Due to the lack of sufficient lasers at the time of performing the experiments, the same ECL used for the Tx AU had to be re-used for the Rx AU. However, since the SFP+ receiver is based on direct detection, no significant penalty is expected to arise when using two independent (i.e. decorrelated) lasers on each unit. After optical amplification, the signal from the IM was filtered with a narrowband optical band pass filter (OBPF) to suppress the upper sideband. The lower-frequency sideband is then sent back to the SFP+ transceiver, where the signal is photo-detected. The LTE analog signal generated after digital processing on the Rx FPGA is connected to a vector signal analyser to measure the error vector magnitude (EVM). A round-trip latency of $2.1 \mu$ s is measured at the Rx FPFGA which is well below the CPRI requirement.

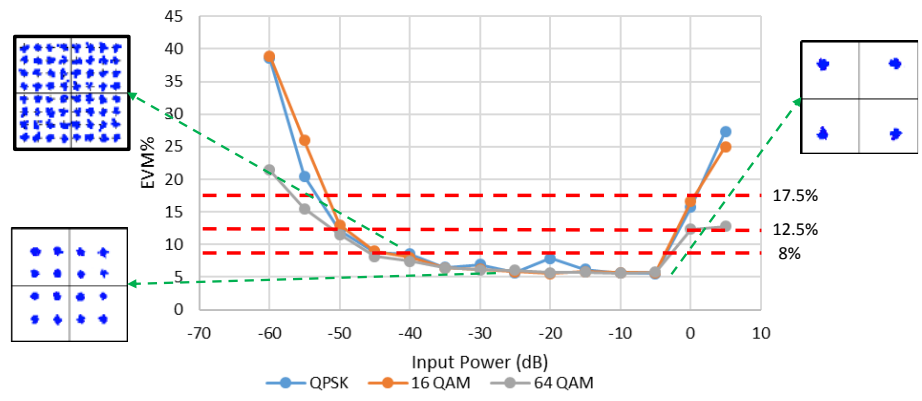

Fig 4. System dynamic range and constellation diagrams

In Fig. 4, the EVM of the LTE signal generated in the Rx FPGA is plotted against the input power from the VSG to the Tx FPGA. Three LTE signals with different modulation formats were tested: quadrature phase shift keying (QPSK), 16-quadrature amplitude modulation (16-QAM) and 64-QAM. As specified by 3GPP, the EVM requirement is $17.5 \%, 12.5 \%$ and $8 \%$ for QPSK, 16-QAM and 64-QAM respectively [7]. The lowest EVM achieved for all the modulation formats is $5.2 \%$ while $1220-\mathrm{MHz}$ LTE replicas are transmitted simultaneously at a line rate of $6 \mathrm{Gbps}$. As can be seen from Fig. 4, dynamic range figures of $40 \mathrm{~dB}$ for 64-QAM, $45 \mathrm{~dB}$ for 16-QAM and $50 \mathrm{~dB}$ for QPSK were obtained.

\section{Conclusion}

This paper demonstrates a new neutral-host RF fronthaul architecture which allows 12-channel digitised LTE compatible services to be transmitted over both optical fibre and $\mathrm{THz}$ links at a fronthaul line rate of $6 \mathrm{Gbps}$ using data compression. It shows that over $40 \mathrm{~dB}$ dynamic range for 64-QAM LTE after transmission can be achieved, with lowest EVM of 5.2\% and round-trip latency of $2.1 \mu \mathrm{s}$. With additional AGC at the analogue frontend, the dynamic range can potentially reach $70 \mathrm{~dB}$ which is sufficient to meet the requirement of MNOs for indoor wireless coverage. $5 \mathrm{G}$ service can be made available by replacing the RF frontend module in the system, which is scalable to support over 10 Gbps data rate encoding $400 \mathrm{MHz}$ spectral bands of RF services.

\section{Acknowledgement}

This work was funded by the UK Engineering and Physical Sciences Research Council Grant Converged Optical \& Wireless Access Networks (COALESCE) EP/P003990/1 and the authors also thank Beijing Institute of Aerospace Control Devices (BIACD) for their support through the SWIFT project.

\section{References}

[1] A. Checko et al., "Cloud RAN for Mobile Networks-A Technology Overview," in IEEE Communications Surveys \& Tutorials, vol. 17, no. 1, pp. 405-426 (2015).

[2] N. J. Gomes and P. Assimakopoulos, "Optical Fronthaul Options for Meeting 5G Requirements," 2018 20th International Conference on Transparent Optical Networks (ICTON) pp. 1-4 (2018).

[3] T. Li, R. V. Penty, and I. H. White, "Novel digital radio over fibre for 4G-LTE," IEEE Int. Conf. Commun. Work. ICCW 312-317 (2015).

[4] H. Shams, T. Li, C. C. Renaud, A. J. Seeds, R. Penty, M. Fice, and I. White, "Digital Radio over Fiber Distribution using Millimetre Wave Bridging," in OFC pp. 1-3 (2018).

[5] T. Li, H. Shams, C. C. Renaud, A. J. Seeds, R. Penty, M. Fice, and I. White, "Multi-service Digital Radio over Fibre System with Millimetre Wave Bridging," Int. Trop. Meet. Microw. Photonics 2018, pp. 1-4 (2018).

[6] S. Rangan, T. S. Rappaport and E. Erkip, "Millimeter-Wave Cellular Wireless Networks: Potentials and Challenges," in Proceedings of the IEEE, vol. 102, no. 3, pp. 366-385 (2014).

[7] 3GPP TS 36.101 V12.6.0, Evolved Universal Terrestrial Radio Access (E-UTRA) User Equipment (UE) Radio Transmission and Reception Release 12 (2014) 